\title{
Zeolite catalysts come into focus
}

\author{
Zeolites have proven to be a boon for industrial catalysis, and our knowledge of how chemical processes work in \\ these materials continues to increase.
}

$\mathrm{t}$ is fair to state that modern society relies on both readily available energy, in the form of liquid fuel, and versatile multifunctional materials, in the form of plastics. The hydrocarbon feedstocks for the synthesis of both of these products can be generated by the fluid catalytic cracking of crude oil, a process that has been used industrially since $1942^{1}$. An alternative synthesis of these hydrocarbon feedstocks is the methanol-to-hydrocarbons (MTH) reaction ${ }^{2}$, which is potentially more environmentally friendly because methanol can be produced from many different sources including biomass. One important class of materials catalyse - that is, speed up - both of these reactions. These are zeolites (pictured), crystalline microporous aluminosilicates that consist of rigid corner-sharing tetrahedral $\left(\mathrm{AlO}_{4}\right)^{5-}$ and $\left(\mathrm{SiO}_{4}\right)^{4-}$ species that assemble together into open framework structures that possess regular pore sizes, enabling easy mass transport, while the acidity can be easily adjusted. This combination of properties has led to their industrial application, as well as fostering interest in their structure and chemistry.

The structure and acidity of zeolites are intimately correlated. Zeolites can possess both catalytically active Brønsted and Lewis acid sites. There are limits on the distribution of tetrahedral aluminium species: they cannot neighbour each other in accordance with Löwenstein's rule (no Al-O-Al bonding). Formally, the $\mathrm{AlO}_{4}$ species are more negatively charged than the $\mathrm{SiO}_{4}$ species, and so a cationic species such as a proton must balance charge, such as a hydroxyl group on a bridging oxygen (Al-OH-Si). This is a Brønsted acid site, with a well-defined structure. Lewis acidity can be induced by doping with other metallic species, or directly in zeolites by high-temperature post-synthesis dealumination that perturbs the aluminium, commonly assumed to be displaced entirely from the framework. However, due to the structural versatility of aluminium these structures are ill-defined. In this issue, a Perspective by Jeroen van Bokhoven and colleagues discusses aluminium Lewis acid sites and structures in zeolites. They argue that, contrary to what is commonly thought, there is no correlation between the number of extra-framework aluminium sites, where aluminium is displaced from its original site, and Lewis acidity, and that the confusion

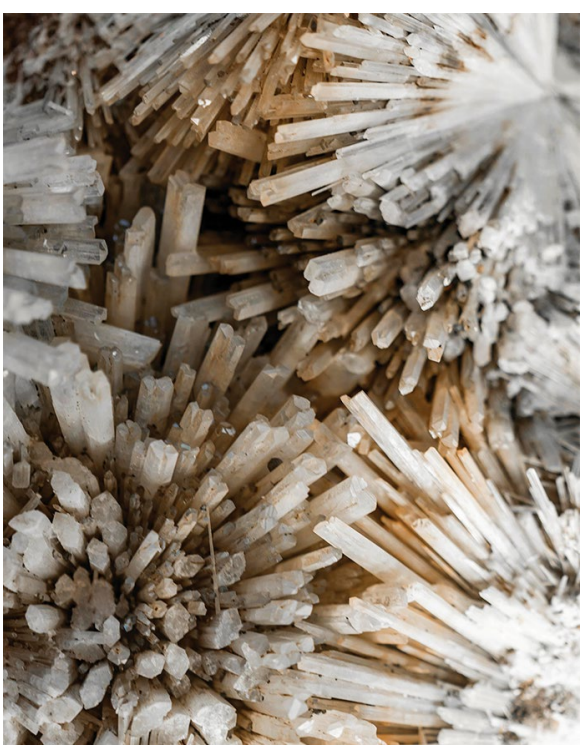

The crystals of a naturally occurring zeolite. Credit: Goran Bogicevic/Alamy Stock Photo

arises due to the different conditions in which Lewis acidity and amount of extra-framework aluminium is determined. As they note, in some cases formation of extra-framework aluminium is not irreversible. They propose that correlating nuclear magnetic resonance and infrared experiments could better identify the strength and location of Lewis acid sites, and emphasize the need for operando experiments to characterize aluminium sites in reaction conditions.

Operando experiments can also shed light into deactivation processes that can occur during catalysis, such as for the MTH reaction where over time 'coking' happens, blocking zeolite pores and so affecting catalytic properties. In an Article by Andrew Beale and colleagues, using Kerr-gated Raman spectroscopy in combination with molecular simulations, the formation of deactivating species in two different zeolites, representative of caged and channel structures, is directly observed. The deactivation process proceeds by formation of extended polyenes that become trapped in the cages and block the pores, these then undergo cyclization to form polycyclic aromatic hydrocarbons that prevent reactant diffusion, hindering activity.

Improving mass transport, and so catalyst stability, can be achieved through increasing the effective surface area by reducing particle size, although the synthesis of zeolite particles less than $100 \mathrm{~nm}$ in size is extremely challenging. In an Article by Jeff Rimer and colleagues, an alternative more facile approach is presented, where nanosized fins are grown on seed crystals. Experimentation and modelling indicates that the mass transport and catalyst properties of the finned zeolites are determined by the fins. The enhanced mass transport, as shown by the reduced molecule residence time, leads to a factor of three increase in catalyst lifetime. As German Sastre notes in the related News \& Views, if even smaller fins can be grown on zeolite particles the surface to volume ratio will further increase, leading to greater enhancement of catalyst properties.

Nucleation and crystallization of zeolites is a fundamental scientific problem. As discussed in a Q\&A with Avelino Corma, other challenges include controlled placement of active sites in specific locations within the zeolite framework, leading to improved catalytic performance, targeted synthesis of a predefined structure, and generation of chiral centres that form enantioselective products. Solving these issues could have direct industrial impact, but for successful collaboration with industry it is often necessary to demonstrate a proof of principle, and any new zeolite application must pass the techno-economic test - that is, the cost and environmental savings balance between new zeolite catalysts and their synthesis.

Zeolites are not limited to petrochemicals catalysis; they can catalyse other feedstock sources such as those from biomass to produce useful chemicals ${ }^{3}$, or selectively reduce toxic compounds such as $\mathrm{NO}_{x}\left(\right.$ ref. $\left.^{4}\right)$. Regardless of whether the application is old or new, increased mechanistic insight into the structure and chemistry of these systems can lead to better materials and processes. Our understanding of zeolites continues to evolve.

Published online: 21 September 2020 https://doi.org/10.1038/s41563-020-00819-x

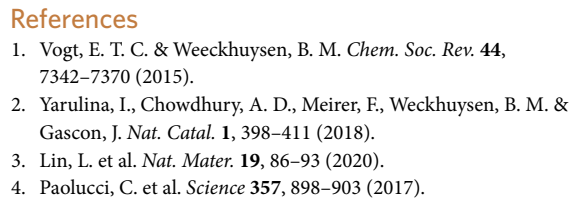

